\title{
Performance of LoRa Network for Environmental Monitoring System in Bidong Island Terengganu, Malaysia
}

\author{
Nur Aziemah Azmi Ali ${ }^{1}$, Nurul Adilah Abdul Latiff ${ }^{2}$, Idrus Salimi Ismail ${ }^{3}$ \\ Faculty of Ocean Engineering Technology and Informatics \\ Universiti Malaysia Terengganu \\ Terengganu, Malaysia
}

\begin{abstract}
Recent wireless communication network which is Low Power Wide Area Network (LPWAN) bring a huge potential in monitoring system as the integration between sensors application and LPWAN technology itself contribute to greater efficiency, higher productivity, and better quality of life in multiform sectors such as in healthcare, smart city application, and monitoring system. The project involves the implementation of Low Power Wide Area Network (LPWAN) while delving into performances of the LoRa Technologies in the development of the environmental monitoring system in Bidong Island located in state of Terengganu, Malaysia. An Arduino Uno microcontroller stacked with LoRa module shield via SPI connection and in conjunction with few sensors work as end device to capture the environment data and send it to the gateway over the long range at a very low-data-rate with low power consumption for remote monitoring system. In this paper, we identify the best spreading factors that works well in the island region by manipulating all the spreading factor from SF6 to SF12. The result shows SF11 provides the best packet delivery ratio and the most stable Receive Signal Strength Indicator (RSSI) compared to other SFs. Moreover, we evaluate the external factors which caused the packet loss data and provide ways to improve the signal quality and to achieve optimal results for signal transmission and communication performance.
\end{abstract}

Keywords-LPWAN; LoRa; environmental monitoring; WSN

\section{INTRODUCTION}

Radio communication with wireless network becomes one of the most life-changing technology trends for these past few decades and it keeps growing with ubiquitous connectivity in every field as everything is interconnected. This interconnection of things refers to the idea of "Internet of Things" (IoT) in smart city applications, intelligent agriculture, smart manufacturing, health-care monitoring system and etc. [1]. Conventional cellular networks and Wi-Fi are the most common networks that offer high data rates and provide the needs of bandwidth-heavy applications such as video streaming and cloud computing to consumers. However, these networks cannot be operated without the main power and are difficult to be deployed in remote and underground areas due to high cost in installation and maintenance. Besides, several issues such as low coverage range, low robustness against network interference and jamming are parts of challenges identified that do not meet some application requirement [2][3][4]. Notwithstanding these limitations, researchers come with a new invention of Low Power Wide Area Network (LPWAN) triggered from Machine-to-Machine (M2M) communication idea to create a communication of information transmission without human intervention.

In this paper, a prototype of an environment monitoring system using LPWAN and LoRa technology was proposed. The environmental sensor had been used to measure the environmental parameters such as temperature and humidity. Then, LoRa performances were analysed in term of signal reliability such as Packet Delivery Ratio (PDR) and Receive Signal Strength Indicator (RSSI). The analysis was done using different spreading factors and different antenna's situation setups at both transmitter and receiver. This paper focuses on the Low Power Wide Area Network which is suitable for a monitoring system in a remote area that does not require high data rates and has minimal power consumptions. Table I presents the comparison between the existing wireless network and LPWAN itself according to [5], [6]. From the tabulated data, it explains the reason why LPWAN was selected to be implemented in this environmental monitoring system.

In the next Section II, we describe the main characteristics of LPWAN and its technologies. Followed by section III; we discuss the related work and in Section IV we explain the methodology used in the development of environmental monitoring system. Then, the results conducted in the experiments are presented and discussed in Section V. Lastly, Section VI concludes this paper. 
TABLE. I. THE CHARACTERISTICS OF EXISTING WIRELESS NETWORK

\begin{tabular}{|l|l|l|l|l|}
\hline $\begin{array}{l}\text { Wireless } \\
\text { System }\end{array}$ & $\begin{array}{l}\text { Long } \\
\text { Range, High } \\
\text { Data Rate } \\
\text { (LTE) }\end{array}$ & $\begin{array}{l}\text { Long } \\
\text { Range, } \\
\text { Low Data } \\
\text { Rate } \\
\text { (LPWAN) }\end{array}$ & $\begin{array}{l}\text { Short } \\
\text { Range, } \\
\text { High Data } \\
\text { Rate (WI- } \\
\text { FI) }\end{array}$ & $\begin{array}{l}\text { Short } \\
\text { Range, } \\
\text { Low Data } \\
\text { Rate } \\
\text { (ZigBee) }\end{array}$ \\
\hline Coverage & Large & Large & Small & Small \\
\hline Range & Long & Long & Short & Short \\
\hline Latency & Low & High & Low & Low \\
\hline Bandwidth & $\begin{array}{l}200 \mathrm{kHz}- \\
900 \mathrm{MHz}\end{array}$ & $\begin{array}{l}500 \mathrm{kHz}- \\
900 \mathrm{MHz}\end{array}$ & $\begin{array}{l}2.4,3.6,5, \\
\text { and 60GHz }\end{array}$ & $<2.4 \mathrm{GHz}$ \\
\hline $\begin{array}{l}\text { Power } \\
\text { Consumption }\end{array}$ & Low & Low & High & Low \\
\hline Operating Cost & Expensive & Cheap & Expensive & Cheap \\
\hline Topology & Star & $\begin{array}{l}\text { Star and } \\
\text { Mesh }\end{array}$ & Star & $\begin{array}{l}\text { Point-to- } \\
\text { point }\end{array}$ \\
\hline Data Rate & High & Low & High & Low \\
\hline
\end{tabular}

II. LOW POWER WIDE AREA NETWORK (LPWAN)

LPWAN arises and complement the conventional networks with the capability to transmit a signal with minimal transmission power about $10 \mathrm{~mW}$ [1]. The transmission of data to the gateway by end device is only few packets per day and it follows below $1 \%$ of duty cycle restrictions set by most country depending on the frequency band used. For instance, Malaysian Communications and Multimedia Commission (MCMC) has set a standard for technical regulatory aspect in a frequency band, maximum power transmit and duty cycle for radio communication devices [7] to be used in Malaysia. Duty cycle in radio communication allows end device to work only when data need to be transmitted or received and it will be turned off if not required. Moreover, LPWAN implies star topology to avoid multiple hops communication and network congestion towards the gateway. The end device transmits the data directly to the gateway resulting in minimal power usage as the end device does not require to listen to the other devices to pass the information. These mechanisms permit the battery to live longer more than 10 years of operation and make it possible to cut down the cost while using an AA and a coin cell batteries for some certain applications [8].

LPWAN offers wide range transmission up to $50 \mathrm{~km}$ and able to cover large areas with a single base station while supporting multiple numbers of connected devices [9]. This is because LPWAN signal propagates in unlicensed sub-1 GHz radio frequency band and adopted unique modulation technique. In Malaysia, radio frequency band of $919-923 \mathrm{MHz}$ is used for LPWAN applications [1]. Sub-1 GHz network band is less congested and less occupied that make it safe from signal interference compared to $2.4 \mathrm{GHz}$ ISM band that is used commonly for Wi-Fi, Cell-Phone peripherals, Microwave ovens, ZigBee, and Bluetooth. Therefore, this Sub-1 GHz band serve a better network propagations for signal performance and more resilient when passing through obstacles by creating more wave diffraction around it and experiences less signal attenuation that allows it to transmit for a longer range [10].
There are two types of modulation technique used by LPWAN technology at the physical layer such as Ultra Narrow Band (UNB) and Spread Spectrum (SS) technique. Sigfox Company [11] uses UNB modulation technique to transmit a very narrow bandwidth signal typically less than $1 \mathrm{kHz}$. This is because it is modulated by passing two significance stages which are known as an abrupt phase shift modulation stage and UNB filtering stage [12]. These stages protocols produce a suitable bandwidth for small uplink traffic and narrowing bandwidth to increase the link budget as the signal power become concentrated [13]. On the other hand, the SS modulation technique adopted by Semtech in LoRa technology [14] spreads the signal energy by reduces the spectral power density into a wide frequency band while preserves the same power intensity. Besides, the signal is secure and clear from traffic and interference as the peak is indistinguishable from the noise signal [15][16].

There are several examples of LPWAN technologies such as LoRa, Sigfox, RPMA [17] and Weightless [18]. However, this project focused on LoRa from Semtech technology. The term LoRa is referred to as "Long-Range" wireless communication system proposed by LoRa Alliance and developed by Semtech for commercial usage. LoRa utilizes Chirp Spread Spectrum (CSS) that has been used in military and space communication to achieve long range and low power energy characteristic [19]. In the CSS scheme, the signal data frequency chipped decreases or increases over a specific time in the form of the chirp signal. The condition of upper-chirp occurs when the frequency changes from lowest to highest, while down-chirp occurs vice versa. This means that the chirp signals have constant amplitude with variable frequency. The technique reduces the design complexity of the receiver as the timing signal and frequency offset between the LoRa transmitter and receiver are equivalent to each other [14]. This situation provides immunity against Doppler Effect and contributes to high receiver sensitivity achieved down to -148 $\mathrm{dBm}$ [20]. Furthermore, the product between high bandwidth and time gives the ability to resist against radio signals band-in or band-out interference while the best selection of broadband chirps able to avoid multipath fading and raise the robustness level. Hence, increasing the maximum link budget up to 157 $\mathrm{dB}$ and lowering the energy consumption by reducing the transmit power [21].

\section{RELATED WORKS}

\section{A. Environmental Monitoring System using Prior Wireless Networks}

Wireless Sensor Network (WSN) has revolutionized the world in many ways. Most of them focus on implementing common wireless networks on their environmental monitoring system. In [22], the authors describe snails monitoring by deploying a ZigBee protocol in a base mote for agricultural plantations and detects the presence of snails by using capacitive sensor to collect the data and pass it to the end device through the router. The research used WSN simulator to design the system and deploy a field test to support the real-life condition. In [23], the authors discussed the issues in Southern Africa on rainfall monitoring system by utilizing the General Pocket Radio Service (GPRS) via cellular network. The data 
are sent directly to a website server for data storage. Authors in[24] propose a new novel of low cost and low power system for smart irrigation specialised in leaf sensing using backscatter radio communication such as in Radio Frequency Identification (RFID) application with $2 \mathrm{~m}$ of communication distance between the tag and the emitter/reader antennas. However, it is preferable if the project implements the IoT system to get easy access of the data. Meanwhile in [25], the authors analysed three different sensor networks for environmental monitoring system using IoT capabilities. One of them employed Wi-Fi communication technology based on User Datagram Protocol (UDP) and Hypertext Transfer Protocol (HTTP) system platforms and the other used power-harvesting on Bluetooth Smart beacon (BLE). This research compared the developed systems in terms of power consumption, network design flexibility, and complexity. The results showed that using WIFI networks to transfer the collected data consumes more energy compared to transfer using the BLE. In addition, data transmitted using WIFI are totally dependable on Internet connection quality where the reduction of signal quality leads to packets loss. On the other hand, to implement BLE in monitoring system, the forwarder device is required to send the collected data from sensors to the cloud.

\section{B. Environmental Monitoring System using LPWAN}

Moving on to the implementation of LPWAN in environmental monitoring system, a few recent studies used LoRa in their project such as in [26]. The authors proposed the idea for a water monitoring system based on IoT and LoRa. The system was set up in a farm area from $1 \mathrm{~km}$ to $3 \mathrm{~km}$ between cattleman's house to the barn. The star topology communication has been implemented in this project and it consists of several nodes that detect and sense the water level in trough and sensor hub is used to collect all the data from the nodes and then the data pass to the gateway. HopeRF RFM95 was used for communication module on both side. At node, the controller used was Atmel ATMega328 with a float switch GE-1307 in order to reach low power system for remote area. While at the sensor hub part, Raspberry Pi v.1 type B was used as a controller because it has SPI protocol to communicate with HopeRF RFM95. The float switch in this project represented the condition of water quantity in the trough. The result showed that the longer the distance, the higher the signal power loss during transmission. Meanwhile, the elevation and polarization between hubs and nodes devices gave impacts on transmission quality. Horizontal polarization is the best position for this water monitoring application. Besides, researchers in [27] presented a new approach on smart water grid management using LPWAN technology by Indian communities in Mori village, India. The project aimed to provide real-time information to the villagers and authorities on quality of water supply and implemented alert mechanism to control the water flow either to step up or step down the water locks and at the same time create awareness on flood management. Moreover, residents around the area were able to use optimal quality water for their home and irrigational purpose according to the needs. The project composed of several sensors such as Oxidation Reduction Potential (ORP), $\mathrm{pH}$, salinity level, turbidity, temperature and flow water sensor. Then, the sensors were mounted to LoRa RF Module and Arduino Nano as major components for the entire system. The collected data is then broadcasted to the LoRa Gateway and stored in the cloud. In this pilot project, the LoRa modules transmitted the data to the gateway via single-hop communication with MAC protocol has its own node address while the App_Key specifically identified which sender that the data comes from. The data that have been transmitted from a gateway and forwarded to the Ericsson cloud were then stored in the cloud according to device ID and next it went through an analytical process that can trigger alert that can be sent through SMS or emails. Lastly, residents were able to reach real-time updates from the dedicated website. To conclude, the project explores the potential of LoRa and at the same time solve the problem such as clean water supplies. Additionally, the authors in [28] deployed an environmental monitoring system with wireless self-powered based on LoRa technology. The system was consist of peripheral nodes or known as sensor nodes such as smoke detection (MQ2), alcohol (MQ3), carbon monoxide (MQ7), luminance (TEMT6000), methane(MQ4), humidity and temperature (HDC1080) sensors. The ATMega328 was used as a controller and LoRa RN2483 as a communication module. Besides detecting and acquiring information, peripheral nodes also transmitted the input to the LoRa gateway via LoRaWAN protocol. The gateway then processed the collected information and upload it to the cloud system via GPRS. This information was accessible to everyone through website or mobile application via ThingSpeak.com. The built prototype was also self-powered by solar energy and it was shown that the system was reliable as there was no packet loss detected.

\section{METHODOLOGY}

The installation of monitoring system involves hardware and software components. We conducted several experiment to identify the best practical setup that can enhance the quality of LoRa signal and increase the packet delivery ratio. The measurements were carried out in Bidong Island $\left(5.6186^{\circ}\right.$, $103.0584^{\circ}$ ) with rough terrain topography and only one square kilometer in an area across the South China Sea. The transmitter node was placed $50 \mathrm{~m}$ above the sea water aligned with the receiver node $75 \mathrm{~m}$ from the sea level on a tophill of Bidong Island. Both LoRa nodes are in a slant path direction to minimize the interruption from obstacles that come rough topography and from other devices that might be use the same channel with our monitoring system. The distance between transmitter and receiver was around $1 \mathrm{~km}$ and in near line-ofsight radio link as presented in Fig. 1.

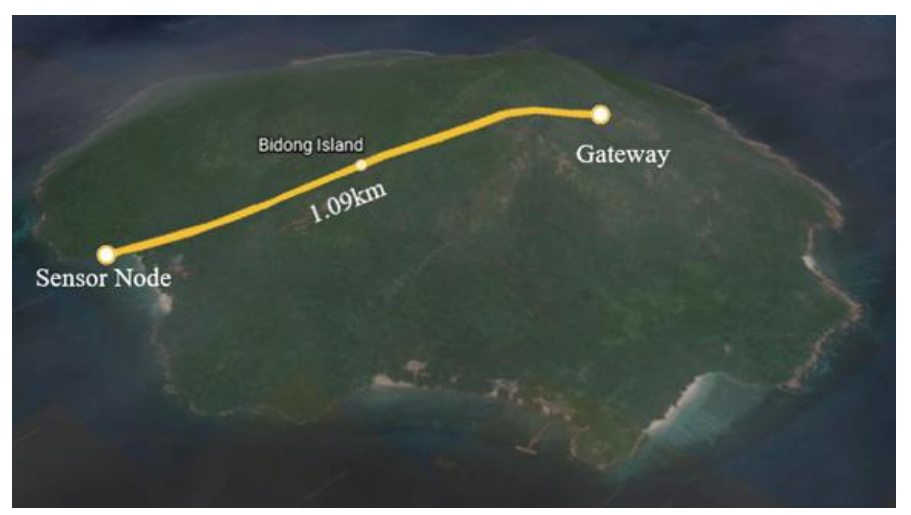

Fig. 1. Location of Experiment in Bidong Island. 


\section{A. System Architecture of Monitoring System}

The system architecture of the developed environmental monitoring system can be simplified into two parts which are LoRa sensor node and LoRa gateway as shown in Fig. 2. LoRa sensor node consists of sensors that functions to collect specific environment data and to define how many time the data must be sent to the gateway. While the gateway will always programmed to receive the data and it became a platform to store the data and allow to remotely monitor and configure the system in the very moment the data has been sent. These platform also enable us to track data loss rate.

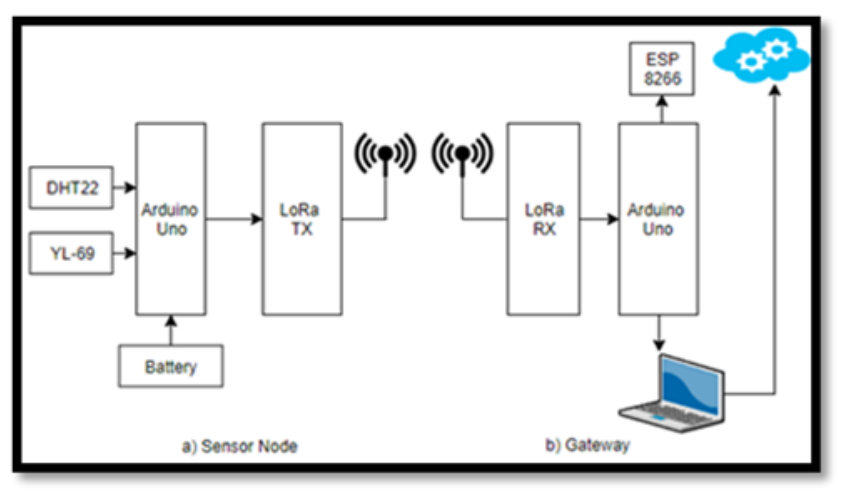

Fig. 2. System Architecture of the Monitoring System.

\section{B. Hardware Component}

The components used in this project composed of sensors, microcontrollers, radio modules, and two types of the antenna as shown in Table II.

\section{Wiring Connection for the Transmitter and Receiver Node}

The wiring connection for the sensor node and the gateway can be seen from the Fig. 3 and Fig. 4,- respectively.

The measurement of environmental parameters equipped with sensors such as temperature and humidity sensor (DHT22) and soil moisture sensor (YL-69) embedded with microcontroller for data acquisition and stack up with LoRa radio module connected all together to form a sensor node before transmit the data to the gateway. Both sensor node and gateway must be connected with correct choice of antenna types with optimal gain value to achieve a certain communication distance either using omnidirectional antenna or Yagi-Uda antenna.

\section{Software Setup}

The research was carried out using the Arduino Integrated Development Environment (IDE) software to write and program the system. The sensor node operated at $5 \mathrm{~V}$ sampled the data from environmental sensors and transmitted to the gateway. The gateway received the data and only the successful packet data transmission displayed at the personal computer and store to a data logger. If the packet was lost, the sensor node will not retransmit the data lost to the gateway instead it skipped the data packet for the next data sensing and transmission. Both sensor node and gateway were set up using the same value of LoRa physical setting to ensure data received via the same channel.
1) LoRa physical setting: LoRa modulation scheme depends on five main configurable setting which are Transmission Power (TP), Spreading Factor (SF), Coding Rate (CR), Bandwidth (BW), and Carrier Frequency (CF). These parameters can be tuned into certain value to meet an optimal condition hence give the best data signal transmission and communication performance.

a) Transmission Power (TP): The power of the propagation signal to transmit a packet in LoRa transceiver and this parameter affects the power expenditure. It can be fine-tuned from $2 \mathrm{dBm}$ to $20 \mathrm{dBm}$ in $1 \mathrm{~dB}$ steps depending on hardware limitation while power usage higher than $+17 \mathrm{dBm}$ limits for $1 \%$ radio duty cycle [29]. Increase the TP will increase the transmission range and data rate.

b) Spreading Factor (SF): The ratio of symbol rate and chip rate. The chip rate is a multiplication of bit rate and $2^{\mathrm{SF}}$ [30] while varying the SF value from SF7 to SF12 can avoid concurrent transmission and does not cause packet collisions because every spreading factors are orthogonal to each other. Higher SFs provide long range but in trade-off low data rate.

c) Bandwidth (BW): The width of a selected frequency used in a transmission band. The transmission of a data sent as a chip rate per second is equal to the bandwidth. LoRa network can be operated in bandwidth either BW125, BW250, and BW500 correspond to $125 \mathrm{kHz}, 250 \mathrm{kHz}$, and $500 \mathrm{kHz}$ respectively. This project used a lower bandwidth as it offer long range transmisson and higher sensitivity.

d) Coding Rate: The factor that increases the protection against corrupted bits in a radio link and known as Forward Error Correction (FEC) rate that allows bits information's recovery. A larger $\mathrm{CR}$ is more reliable and offers more protection but consumes more energy and increase the timeon-air. CR can be select either $4 / 5,4 / 6,4 / 7$ or $4 / 8$. LoRa radio always used CR $4 / 8$ for the payload stored in the header packet and if being used as an explicit header the radios with different $\mathrm{CR}$ are still able to communicate with each other.

e) Carrier Frequency: The central frequency band that transmit the signal and depends on the allowed ranges of local regional frequency. For instance in Malaysia, Malaysian Communication Multimedia Commission (MCMC) has set a specific frequencies band for radio communication devices to be operated only between frequencies of $919 \mathrm{MHz}$ to $923 \mathrm{MHz}$. While, in Europe frequency band available were $433 \mathrm{MHz}$ and $868 \mathrm{MHz}$.

The whole experiment was conducted based on LoRa Physical setting as tabulated in Table III.

\section{E. Experimental Setup}

An antenna is a conductor that converts high-frequency alternating current $(\mathrm{AC})$ signals to radio frequency signals and radiates into the air to form radio waves. In this experiment, two types of antennas were used to be connected with the LoRa module which $5 \mathrm{dBi}$ gain omnidirectional antenna was shown as in Fig. 5 and $11 \mathrm{dBi}$ gain the Yagi-Uda antenna as shown in Fig. 6 while varying the situation of the transmitter and the receiver at both locations as in Table IV. 
TABLE. II. LIST OF COMPONENT USED IN THIS EXPERIMENT

\begin{tabular}{|c|c|}
\hline Component & Explanation \\
\hline $\begin{array}{l}\text { DHT22 Temperature Sensor } \\
\text { Module }\end{array}$ & $\begin{array}{l}\text { This 3-pins small size sensor measures the } \\
\text { relative humidity and temperature values of } \\
\text { its surrounding. It consumes only } 0.3 \mathrm{~mA} \\
\text { when operating and } 60 \mu \mathrm{A} \text { when on } \\
\text { standby. Able to withstand severe } \\
\text { application types with great reliability and } \\
\text { stability. It has an accuracy of } \pm 1 \% \mathrm{RH} \text { for } \\
\text { humidity with range } 0 \% \text { to } 100 \% \text { and for } \\
\text { the temperature range of }-40^{\circ} \mathrm{C} \text { to } 80^{\circ} \mathrm{C} \\
\text { with } \pm 0.5^{\circ} \mathrm{C} \text { of accuracy. }\end{array}$ \\
\hline YL-69 with LM393 & $\begin{array}{l}\text { This simple sensor was used to measure the } \\
\text { soil moisture and it comes with two parts } \\
\text { which are the probes and the electronic } \\
\text { module. Operates at voltage } 3.3 \text { to } 5 \mathrm{~V} \text { and } \\
\text { able to tuning the sensitivity via digital } \\
\text { potentiometer. When the moisture in the } \\
\text { soil is high, the output voltage decreases, } \\
\text { while if the moisture is low, the output } \\
\text { voltage increases. }\end{array}$ \\
\hline Arduino Uno & $\begin{array}{l}\text { ATmega328p was selected as a } \\
\text { microcontroller module on the Arduino } \\
\text { Uno to control the sensors and it was } \\
\text { embedded with communication module } \\
\text { since it has the capability to convert 10-bit } \\
\text { analog-to-digital (ADC) and has high- } \\
\text { performance feature such as } 16 \text { clock speed } \\
\text { and consists of } 14 \text { digital I/O pins that } \\
\text { allows various type of application with low } \\
\text { cost and low power consumption. }\end{array}$ \\
\hline 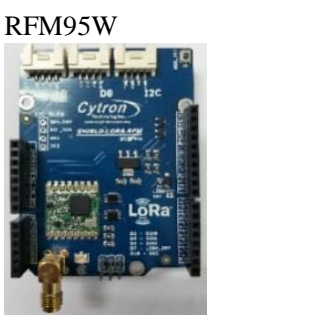 & $\begin{array}{l}\text { LoRa module featured with SX1278 } \\
\text { transceivers as a communication module } \\
\text { and able to operate well with the } \\
\text { microcontroller via Serial Peripheral } \\
\text { Interface bus (SPI). LoRa can reach long } \\
\text { distance communication and the data } \\
\text { transmission is robust through their chirp } \\
\text { spread spectrum and this module operates } \\
\text { in the } 915 \mathrm{MHz} \text { frequency band and has - } \\
146 \mathrm{dBm} \text { receiver sensitivity. }\end{array}$ \\
\hline Omni-Directional Antenna & $\begin{array}{l}\text { This high performance of } 5 \mathrm{dBi} \\
\text { omnidirectional antennas operates in } \\
\text { ranges between } 868 \mathrm{MHz} \text { to } 915 \mathrm{MHz} \text { of } \\
\text { ISM band is ideal for multipoint and non- } \\
\text { line-of-sight radio link. Connected with } \\
\text { SMA Male copper connector and works in } \\
\text { vertical polarization. }\end{array}$ \\
\hline 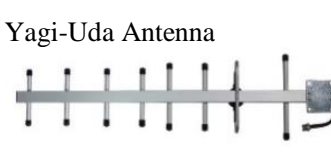 & $\begin{array}{l}\text { A powerful } 11 \mathrm{dBi} \text { gain Yagi-Uda } \\
\text { directional antenna able to create strong } \\
\text { focusing signal in one direction for } \\
\text { maximum signal strength and performance } \\
\text { over the long distances. }\end{array}$ \\
\hline
\end{tabular}

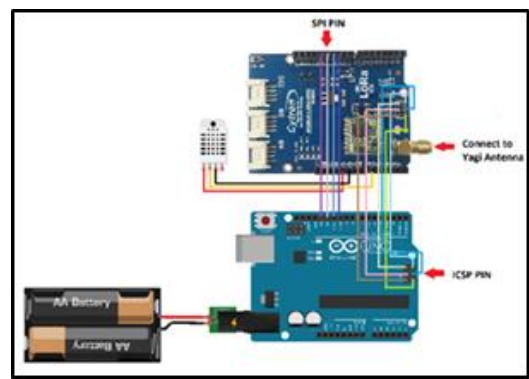

Fig. 3. Sensor Node act as LoRa Transmitter.

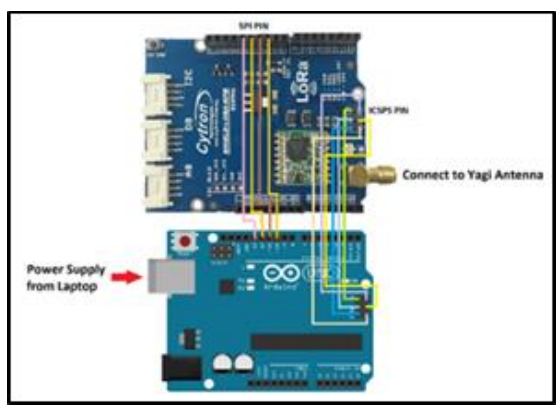

Fig. 4. Gateway Act as LoRa Receiver.

TABLE. III. LORA PHYSICAL SETTING USED IN THIS EXPERIMENT

\begin{tabular}{|c|l|l|l|c|l|}
\hline TP & SF & BW & CR & CF & Payload \\
\hline $23 \mathrm{dBm}$ & $7-12$ & $125 \mathrm{kHz}$ & $4 / 8$ & $919 \mathrm{MHz}$ & 5 Bytes \\
\hline
\end{tabular}

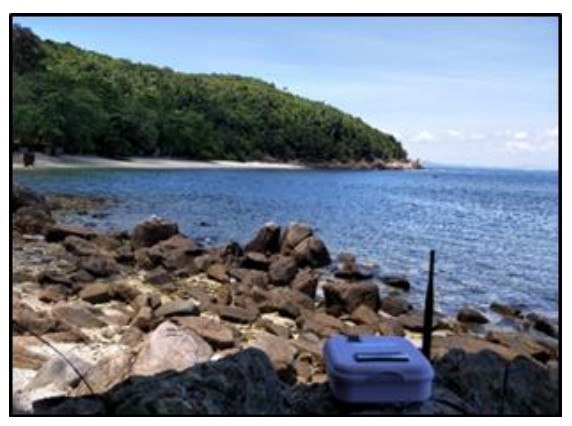

Fig. 5. The Sensor Node Connected with Omnidirectional Antenna.

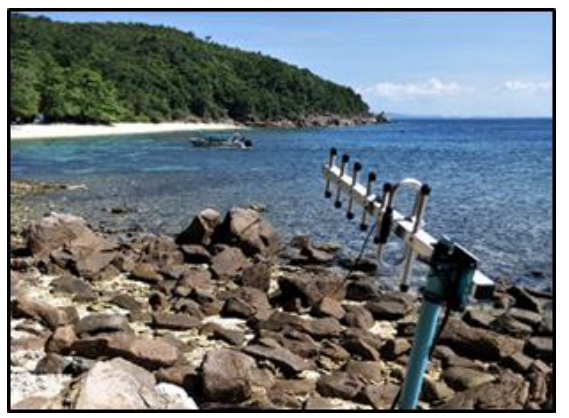

Fig. 6. The Sensor Node Connected with Yagi-Uda Antenna.

TABLE. IV. ANTENNA TYPE AND SET UP FOR LORA NODES

\begin{tabular}{|l|l|l|}
\hline Types of Antenna & Transmitter & Receiver \\
\hline Situation A & Omni-directional & Omni-directional \\
\hline Situation B & Yagi-Uda & Yagi-Uda \\
\hline Situation C & Yagi-Uda & Omni-directional \\
\hline Situation D & Omni-directional & Yagi-Uda \\
\hline
\end{tabular}

\section{RESUlT AND DisCUSSION}

\section{A. Signal Quality Performance}

The device prototype has been developed and the results define the quality of signal transmission over the LoRa physical layer by using different spreading factors in different situations of antennas. The spreading factor was varied from SF7 to S12 and has been set up according to the situations of antenna mentioned in Table IV of this experiment. 
From the Fig. 7, SF11 is noticeable to give better reliability among other SFs and works well in every situation of the antenna including in situation A where the omnidirectional antenna was used for both transceiver nodes and also in situation $\mathrm{D}$, where the omnidirectional used as a transmitter and received by Yagi-Uda antenna. The value of RSSI is slightly affected by varying the spreading factor but mostly affected by the changes in the antenna type and antenna gain as well as transmission power. The whole experiment was decided to use $23 \mathrm{dBm}$ of transmission power (TP) because lower TP cannot transmit the data packet in this type of nonline of sight (NLOS) radio link. This result proved that to increase the signal quality, it is crucial to set up the transmitter and receiver in the most suitable situation with suitable LoRa PHY layer and also by considering the surrounding obstacles.

The types of the antenna are also an important factor to transmit or receive data. In this experiment, we observed that Yagi-Uda antennas provide more reliable data compare to omnidirectional antenna because it transmits and receives signals directly from a specific direction with a concentrated and strong signal beam. Meanwhile, the omnidirectional antenna radiates or receives the signal uniformly from all directions in 360 degrees horizontally with equal signal power. For that reason, the result obtained in this experiment might be slightly affected by the unmatched polarization between the transmitter and receiver position. The orientation of the antenna's nodes that we fixed depends on our smartphone's compass application and the calibration can be slightly inaccurate due to metal and other electronic interfered.

Next, Fig. 8 presents the graph of Signal-to-Noise Ratio (SNR) while Fig. 9 presents the graph of the Packet Delivery Ratio (PDR) and both are over the Spreading Factor (SF). The results show it is possible to increase the rate of PDR and the range of communication by using the higher SF (e.g.; SF12). However, it seems that increased SF caused more packets collision due to longer transmission time and enhanced the Signal-to-Noise Ratio (SNR) and increased the data lost. While it is also possible to use lower SF (e.g.; SF7) but, we must consider the link budget and the type the antenna for optimum signal transmission in a particular range.

\section{B. External Factors that affect Signal Performance}

The results obtained also correlated with Free Space Path Loss (FSPL) factor between two antennas. FSPL is a power loss at a rate inversely proportional to the distance travelled and proportionally to the wavelength of the signal illustrated as in Fig. 10.

$F S P L=20 \log _{10}\left[\frac{4 \pi d}{\lambda}\right]\{d B\}$

The calculation of path loss theory for every situation is recorded as in Table $\mathrm{V}$ below by referring to the equation (1). The value of FSPL for both situations in C and D is the same, but the result indicated that the antenna setup in situation $\mathrm{C}$ is more reliable compared to the situation in D because as mentioned in the previous section Yagi-Uda antenna that acts as transmitter radiates a strong signal and gives ability to directs the signal in a long distances and it is easier to the receiver to catch that signal compared to situation $\mathrm{D}$ where omnidirectional acts as a transmitter. The signal power from the omnidirectional antenna is not strong enough to be caught by the receiver.

Another important factor that contributes to this phenomenon is the Fresnel zone between transmitter and receiver. The Fresnel zone is a concentric ellipsoid-shaped area around the LOS path as shown in Fig. 11 and the radius of the Fresnel zone in this experiment is 9.03 meters calculated as in equation (2). The equation refers to the radius of the zone at its widest point where the signals propagate after leaving the antenna. Almost $40 \%$ of the Fresnel zone at the receiver location is blocked by the obstacle such as growing trees, old water tank, branches, plant canopy, and shrubs nearby the hilltop of Bidong Island. This obstruction affects the signal propagation by inducing the multi-path issues as the signal will be reflected, refracted, and diffracted away from the receiver and causing signal degradation and loss.

Radius $(m)=17.31 \times \sqrt{\frac{\text { Distance }(\mathrm{km})}{4 f(\mathrm{GHz})}}$

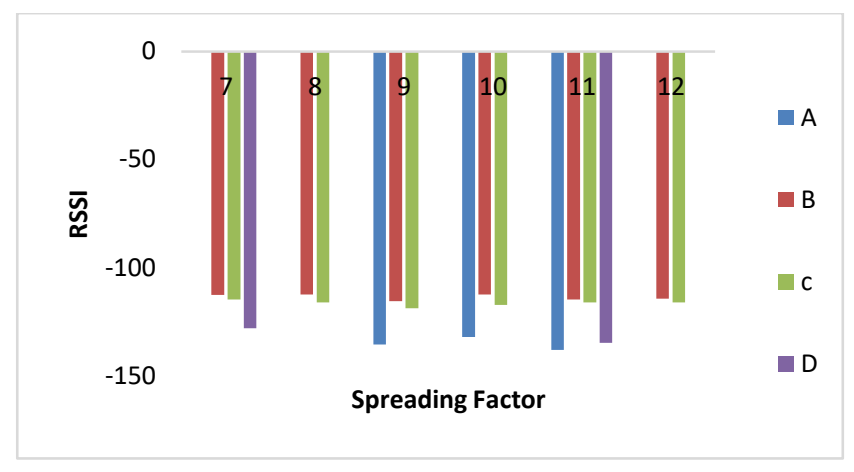

Fig. 7. Graph of RSSI Varies with different SF and different Antenna Situation.

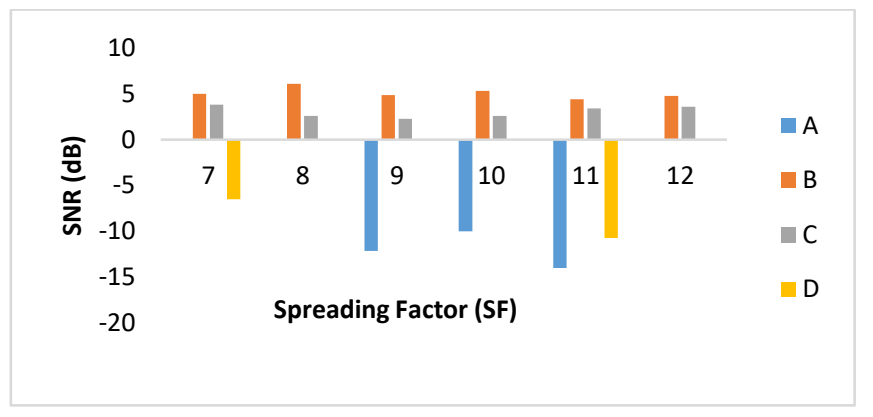

Fig. 8. Graph of SNR Varies with different SF and different Antenna Situation.

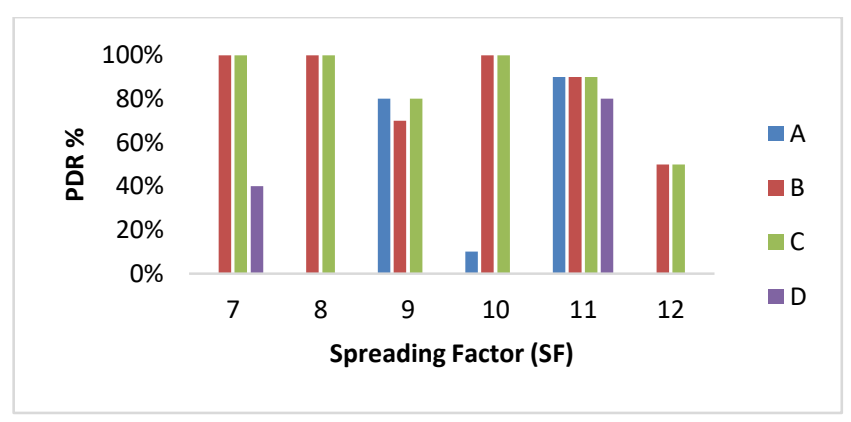

Fig. 9. Graph of PDR Varies with different SF and different Antenna Situation. 


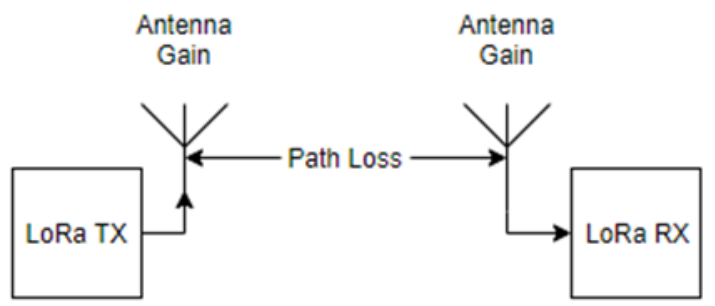

Fig. 10. Block Diagram of Free Space Path Loss.

TABLE. V. CALCUlation OF FSPL

\begin{tabular}{|l|l|}
\hline Situation & FSPL $(\mathbf{d B})$ \\
\hline A & 81.71 \\
\hline B & 69.71 \\
\hline C & 75.71 \\
\hline D & 75.71 \\
\hline
\end{tabular}

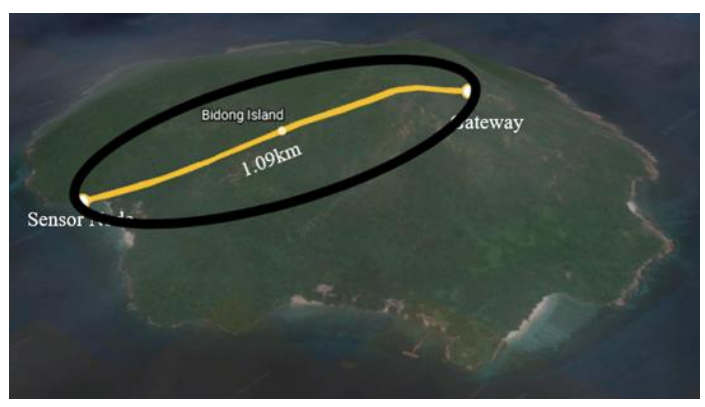

Fig. 11. The Fresnel Zone of Experimental Location.

\section{CONCLUSION}

The paper has presented the hardware and software structures of the monitoring system and the details of each component used. The project also highlighted the implication of antenna settings as its effect on the signal transmission and explained the significance of selection in LoRa physical layer settings. The results of this research support the idea that YagiUda directional antenna is the best choice for long-distance signal propagation that radiates strong beams transmission compared to an omnidirectional antenna. Meanwhile, dealing with the right antennas gains and polarization can lower the data rate loss and increase the signal quality. Besides, increase the antenna height for both transmitter and receiver also improves the signal propagation because it can reduce the ground refraction. Another important practical implication is to clear the Fresnel zone from any signal interferer and blockage that leads to signal loss such as growing trees, shrubs, and branches. Besides, one of the findings of the project was the value of the spreading factor that can be used in most of the situations is SF11 because of its variability. This is proven that with a suitable selection of SF can greatly impact the result of LoRa performances and improves the maximum range of coverage. Lastly, future research might explore the cloud server for the IoT system to be applied using ThingSpeak or any other open clouds platform for online processing data storage and analytics system.

\section{ACKNOWLEDGMENT}

The authors would like to be obliged to Luimewah (M) Sdn Bhd because of the project assistance throughout this project progress and this research was supported by University Malaysia Terengganu (UMT) and Ministry of Education Malaysia with financial assistance under project no. 59466.

\section{REFERENCES}

[1] MCMC, Iot-Technical-Regulatory-Aspects-Key-Challenges-180406014 354.Pdf..

[2] A. Lavric and V. Popa, "A LoRaWAN: Long Range Wide Area Networks Study," pp. 417-420, 2017.

[3] X. Vilajosana, F. Adelantado, X. Vilajosana, P. Tuset-peiro, B. Martinez, and J. Melia, "Understanding the limits of LoRaWAN Understanding the limits of LoRaWAN," no. July, 2016.

[4] I. S. Ismail, "Performance analysis of data recovery via application layer for LPWAN," 2019 IEEE 89th Veh. Technol. Conf., pp. 1-5, 2019.

[5] M. M. Alsulami and N. Akkari, "The role of 5G wireless networks in the internet-of- things (IoT)," 2018 1st Int. Conf. Comput. Appl. Inf. Secur., pp. 1-8, 2018.

[6] J. E. Shuda, A. J. Rix, and M. J. Booysen, "Towards Module-Level Performance and Health Monitoring of Solar PV Plants using LoRa Wireless Sensor Networks," 2018 IEEE PES/IAS PowerAfrica, pp. 172177, 2018.

[7] M. Communications and M. Commission, "CLASS ASSIGNMENT NO . 1 OF 2017," no. 1, 2017.

[8] U. Raza, P. Kulkarni, and M. Sooriyabandara, "Low Power Wide Area Networks: An Overview," IEEE Commun. Surv. Tutorials, vol. 19, no. 2, pp. 855-873, 2017.

[9] E. De Poorter et al., "Sub-GHz LPWAN Network Coexistence, Management," 2017.

[10] S. Aust, "Analysis of the Performance Boundaries of Sub-1 GHz WLANs in the $920 \mathrm{MHz}$ ISM-Band," pp. 1-5.

[11] Sigfox, "Sigfox Technical Overview," vol. 1, no. May, p. 26, 2017.

[12] X. Xiong, K. Zheng, R. Xu, W. Xiang, and P. Chatzimisios, "Low power wide area machine-to-machine networks: Key techniques and prototype," IEEE Commun. Mag., vol. 53, no. 9, pp. 64-71, 2015.

[13] M. Anteur, V. Deslandes, N. Thomas, and A. L. Beylot, "Ultra narrow band technique for low power wide area communications," 2015 IEEE Glob. Commun. Conf. GLOBECOM 2015, pp. 1-6, 2015.

[14] Semtech, "LoRaTMModulation Basics Semtech," 2015.

[15] "Understanding Spread Spectrum for Communications - National Instruments." [Online]. Available: http://www.ni.com/en-my/innovations/ white-papers/06/understanding-spread-spectrum-forcommunications.html. [Accessed: 28-Apr-2019].

[16] S. Kartakis, B. D. Choudhary, A. D. Gluhak, L. Lambrinos, and J. A. McCann, "Demystifying low-power wide-area communications for city IoT applications," pp. 2-8, 2016.

[17] “Technology - Ingenu." [Online]. Available: https://www.ingenu.com/ technology/. [Accessed: 13-May-2019].

[18] "What is Weightless - Weightless." [Online]. Available: http://www. weightless.org/about/what-is-weightless. [Accessed: 13-May-2019].

[19] LoRa Alliance, “A technical overview of LoRa ${ }^{\circledR}$ and LoRaWAN TM LoRaWAN TM What is it?," no. November, 2015.

[20] “'Sx1276/77/78," no. July, p. 2012, 2012.

[21] K. Mikhaylov, M. Pettissalo, and J. Peta, "Performance of a low-power wide-area network based on LoRa technology: Doppler robustness , scalability , and coverage," vol. 13, no. 3, 2017.

[22] D. García-lesta, D. Cabello, E. Ferro, P. López, and V. M. Brea, "Wireless Sensor Network With Perpetual Motes for Terrestrial Snail Activity Monitoring," vol. 17, no. 15, pp. 5008-5015, 2017.

[23] E. M. Mangundu, J. N. Mateus, G. L. Zodi, and J. Johson, “A Wireless Sensor Network for Rainfall Monitoring, using Cellular Network: A Case for Namibia," pp. 240-244, 2017. 
[24] S. N. Daskalakis, G. Goussetis, S. D. Assimonis, M. M. Tentzeris, and A. Georgiadis, "A uW Backscatter-Morse-Leaf Sensor for Low-Power Agricultural Wireless Sensor Networks," IEEE Sens. J., vol. 18, no. 19, pp. 7889-7898, 2018.

[25] G. Mois, S. Folea, and T. Sanislav, "Analysis of Three IoT-Based Wireless Sensors for Environmental Monitoring,” IEEE Trans. Instrum. Meas., vol. 66, no. 8, pp. 2056-2064, 2017.

[26] Lukas, W. A. Tanumihardja, and E. Gunawan, "On the application of IoT: Monitoring of troughs water level using WSN," 2015 IEEE Conf. Wirel. Sensors, ICWiSE 2015, no. August 2015, pp. 58-62, 2016.
[27] M. Saravanan, A. Das, and V. Iyer, "Smart water grid management using LPWAN IoT technology," GIoTS 2017 - Glob. Internet Things Summit, Proc., 2017.

[28] K. Tzortzakis, K. Papafotis, and P. P. Sotiriadis, "Wireless Self Powered Environmental Monitoring System for Smart Cities based on LoRa," 2017 Panhellenic Conf. Electron. Telecommun., 2018.

[29] M. Bor and U. Roedig, "LoRa transmission parameter selection," Proc. 2017 13th Int. Conf. Distrib. Comput. Sens. Syst. DCOSS 2017, vol. 2018-Janua, no. June, pp. 27-34, 2018.

[30] M. H. Habaebi, I. J. Chowdhury, M. R. Islam, and N. A. B. Zainal, "Effects of shadowing on LoRa LPWAN radio links," Int. J. Electr. Comput. Eng., vol. 7, no. 6, pp. 2970-2976, 2017. 